perpendicular to and slightly skewed with respect to the filament axis. Using data from the literature, the authors were able to identify the pointed end of the filament which determines the direction in which actin is translocated by myosin and locate the myosin binding site on the large domain of the actin monomer. In the filament, each actin subunit interacts with four of its neighbours and the contacts seem to involve all four subdomains. The most extensive interactions between subunits are made axially (along each of the twin actin strands rather than between them). The model explains many of the results from chemical and spectroscopic measurements - for example the accessibility of lysine residues - thus lending further credibility to its general correctness. Using data from the literature, the authors were able to identify the pointed end of the filament which determines the direction in which actin is translocated by myosin.

In these two papers, though, the authors refer to the atomic structure of the actin:DNase I complex as well as to the atomic model of the actin filament. Are these not both atomic models whose correctness can be judged by their fit to X-ray data? The distinction here lies in the certainty with which the atoms can be located. With a 2.8 - $\AA$-resolution map obtained by isomorphous replacement, the positions of atoms can be determined to about $0.5 \AA$. In producing a model of a filament, however, the authors assumed that the subunit conformation as seen in the complex remains unchanged in the filament. This is the proper first approximation to adopt, and indeed, the model agrees reasonably well with the $\mathrm{X}$-ray data and seems to explain many other observations. In the next step, the authors have begun to refine the model by allowing the subdomains to move relative to one another. In so doing one of the subdomains has shifted by 15 degrees. Thus, as refinement proceeds, significant changes in atomic positions could occur that would affect the details of the interatomic interactions, although the broader features of the filament model will be unaffected.

The atomic model, although the most detailed, is not the only model of the actin filament. There are various maps and models derived from electron micrographs of either filaments on their own or with accessory proteins, such as myosin. All these views reveal differences that have been the source of much debate. Will the X-ray model now take precedence? The important caution is that observed differences between models may result from real differences in structure: for example, the presence of myosin or its S1 fragment might alter the structure of actin significantly.

Moreover, actin not only binds ATP but also catalyses its hydrolysis to ADP. The role of this reaction is intriguing, because the energy released by the reaction could be used to alter the conformation of actin. Indeed, P. A. Janmey and colleagues find that ADP-actin filaments may adopt two conformational states. On page 95 of this issue, these authors report that ADP-actin filaments made by hydrolysis of ATPactin filaments are rigid whereas those filaments made by polymerizing ADPactin subunits are flexible. They suggest that in the hydrolysis of ATP to ADP, energy is trapped and stored in the filaments. Such energy would be available for mechanical work in the cell.

The notion that actin filaments might perform work in a cell in the absence of myosin seems heretical given the exciting recent results on myosin motors (see $\mathbf{J}$. A. Spudich, Cell Regulation 1, 1-11; 1989). In the formation of the acrosomal process in marine sperm cells, however, the polymerization of actin or the uncoiling of an actin bundle can generate motion without any evidence of help from myosin, although other proteins are involved. The movement is quite dramatic: a 60-90-micrometre-long cell extension shoots out at about 10 micrometres per second. In the test tube, even in the absence of other proteins, the actin filament can vary its structure. These changes have been variously explained as angular disorder, lateral slippage of the two substrands and a change in flexibility. It is unclear what causes these changes or indeed whether they are all manifestations of the same phenomenon.

Holmes, Kabsch and colleagues have compared the structure of the ADPactin-DNase complex with that of the ATP-actin complex but see no differences except, of course, for the additional phosphate. Perhaps this is not unexpected, because the presence of DNase inhibits the ability of the actin subunit to exchange bound nucleotide with nucleotide in the medium. The authors point out that the DNase molecule sits on top of the nucleotide cleft, where it can lock the cleft in one conformation and might also prevent any changes resulting from nucleotide exchange. By contrast, actin has an enhanced ability to exchange bound nucleotide when complexed with the protein profilin. Profilin sits at the opposite end of the actin subunit, at the bottom of the cleft, and could therefore hold the cleft in a different, open conformation. Fortunately, elucidation of the structure of the actin-profilin complex by C. E. Schutt and his colleagues at Princeton is nearing completion: we might soon be treated to a view of the actin subunit in one of its other conformations.

David J. De Rosier is at the Rosentiel Center, Brandeis University, Waltham, Massachusetts 02254-9110, USA.

\section{Water at bay}

How does the non-stick coating stick to the frying pan? Not very well, is the usual answer; a firmly adhering non-stick coating is almost a technical contradiction. But Daedalus has a way out. $\mathrm{He}$ is anxious to exploit graphite fluoride, the non-stickiest, most unwettable material of all; and plans to form it as an integral chemical part of the surface to be coated.

Most organic materials can be decomposed to graphite by simple heating: this is how carbon fibre is made. Ultra-rapid laser-pulse irradiation can raise the surface of a solid to a high temperature while leaving the interior quite cold. So DREADCO's physicists are scanning lasers across various solid polymeric samples, decomposing their surfaces to a thin layer of graphite still chemically bonded to the material beneath. Subsequent treatment with fluorine should then give the ultimate non-stick, non-wet, firmly attached graphite fluoride surface coating.

DREADCO's first 'Grafluorinated' products will be plastic windows and lenses. Their novel surface 'bloom' will reduce reflections, but will also be uniquely water repellent. Goggles and spectacles with the new lenses will never mist up. Droplets condensing on them will be unable to adhere and will simply slide down. Raindrops, instead of giving the curious splotched and haloed outlook known to all spectacle wearers, will just bounce off. Windscreens made of the new material will need no wipers, and windows made of it will never need cleaning. Unable to adhere, dirt will just blow away; even frost will slide helplessly off.

In the same way, a Grafluorinated paint surface would never hold the dirt, and would be so water repellent that the metal underneath could never rust. The implications for the car industry are truly awesome, and those for marine engineering even more so. A true non-stick non-wet ship's paint should repel barnacles and other fouling organisms totally, giving wonderfully low drag. Even better, imagine a hairy carbon-fibre coating, flock-sprayed onto the ship's surface and fluorinated in situ. The unwettable fibres would retain the air between them as a thin layer, and the boat would hardly touch the water at all. Like the water-vole (whose water-repellent coat works a similar trick) it would skim along on a thin layer of lubricating air, saving a vast amount of fuel. Similar non-wet internal coatings for pipes and hoses should transform the pumping efficiency of the water industry, the fire-fighting services and chemical engineering generally. And Grafluorinated clothes would repel water perfectly even if you fell in, while being so dirt proof that you could simply shake them clean.

David Jones 